\title{
Highly selective photoenhanced wet etching of GaN for defect investigation and device fabrication
}

\author{
P. Visconti ${ }^{1}$, M. A. Reshchikov, K. M. Jones, F. Yun, D. F. Wang, R. Cingolani and \\ H. Morkoç \\ Virginia Commonwealth University, Dept. of Electrical Engineering, Richmond, VA, 23284 \\ ${ }^{1}$ Also with Istituto per lo Studio di Nuovi Materiali per l'Elettronica, CNR, Lecce, 73100, ITALY \\ C. W. Litton \\ Air Force Research Laboratory, Wright Patterson AFB, OH 45433 \\ R. J. Molnar \\ Massachusetts Institute of Technology, Lincoln Laboratory, Lexington, MA, 02420-9108
}

\begin{abstract}
Photoenhanced electro-chemical (PEC) wet etching has been shown to be suitable for dislocation-density estimation in $\mathrm{n}-\mathrm{GaN}$ films as well as for $\mathrm{GaN}$-based device fabrication. We report on PEC etching of $\mathrm{n}-\mathrm{GaN}$ samples grown by MBE and HVPE methods in unstirred aqueous $\mathrm{KOH}$ solution under $\mathrm{He}-\mathrm{Cd}$ laser illumination. Characterization of the etched samples was carried out using atomic force microscopy (AFM) in both cross-sectional and plan-view configurations and scanning electron microscopy (SEM). At moderate illumination densities, the SEM and AFM analyses reveal sub-100 $\mathrm{nm}$ scale threading vertical wires on the etched surfaces. The calculated density $\left(\sim 1 \times 10^{9} \mathrm{~cm}^{-2}\right)$ is in agreement with dislocation density found by transmission electron microscopy. Using cross-sectional AFM, we find that these vertical wires are $\sim 1 \mu \mathrm{m}$ high and are perpendicular to the sapphire surface. Applying a higher illumination density or an external voltage, we obtain a higher etch rate with a smooth free-feature etched surface. Some highly resistive samples that cannot be etched under normal conditions because the band bending is too small to confine the holes to the surface for them to participate in the PEC process, can be etched with the application of a voltage to the sample. In this case, the etch rate depends on both the polarity and the magnitude of the voltage applied. In an MBE-grown sample with an AlN/GaN superstructure inside, we report on high selectivity between AlN and $\mathrm{GaN}$ (AlN is an etch stop); the selectivity is due to the etching mechanism of the PEC process.
\end{abstract}

\section{INTRODUCTION}

Nitride materials are distinguished by their chemical stability, a characteristic that has required unique challenges for device fabrication [1,2]. Most processing of III-nitrides is conducted by dry plasma etching despite the resulting ion-induced damage and difficulty in obtaining smooth etched sidewalls as required for devices such as lasers $[2,3,4,5,6]$. No wet solution has been found to etch significantly the Ga-face of epitaxial $\mathrm{GaN}$ at room temperature. Elevation of the temperature $\left(>120^{\circ} \mathrm{C}\right)$ can increase the etch rates but the difficulties associated with finding an effective mask layer make this process unsuitable for nitride-based device processing. Thus, there have been several attempts to utilize assisted wet-etching techniques. Photo-enhanced electro-chemical (PEC) etching has been demonstrated to produce high etch rates and anisotropic etch profiles in GaN films at room temperature [7, 8.9, 10,11]. Compared to dry etching processes, PEC etching has the advantage of low surface damage, low equipment cost and simplicity. 
Additionally, Youtsey et al. demonstrated PEC process for dislocation density estimation in n-doped $\mathrm{GaN}$ films $[12,13,14]$. They reported nanometer-scale free-standing whisker-like features obtained by selectively etching $\mathrm{GaN}$ between dislocation sites under very precise etching conditions. With cross-sectional transmission electron microscopy (TEM), they showed the presence of both pure-edge and mixed pure-screw dislocations in the whiskers. Dislocation sites have been modeled as negatively charged Coulomb centers becoming sinks for photogenerated holes [15]. Therefore, the spatially variable surface concentration of holes leads to a reduced etch rate at dislocation sites, in turn resulting in whisker formation.

The characterization of dislocations in nitride films is carried out principally using planview and cross-sectional TEM, which require extensive sample preparation. Because the PEC etching procedure is significantly simpler and less time consuming, it can be an excellent precursor to TEM analysis for defect characterization.

We carried out PEC etchings of $\mathrm{n}-\mathrm{GaN}$ samples in unstirred aqueous $\mathrm{KOH}$-based solution under He-Cd laser illumination. At moderate illumination densities and under slightly carrierlimited conditions, the selective PEC process produces nanometer-scale whisker-like vertical features on the etched surfaces. Using similar etching conditions and by applying an external bias, or alternatively using high illumination density, we obtain a high etch rate with free-feature smooth surfaces. Highly resistive GaN samples cannot be etched under normal etching conditions because the band bending at the surface is too small to confine holes. However, the application of a single-polarity voltage allows etching to occur. Finally, we also report on high selective etching in a sample containing an AlN/GaN superstructure; in this case, the AlN thin layer was the stop layer.

\section{EXPERIMENTAL DETAILS}

Three different sets of $\mathrm{GaN}$ samples were used for the PEC experiments. The first were intentionally Si-doped n-type $\left(\mathrm{n} \approx 2 \times 10^{18} \mathrm{~cm}^{-3}\right) \sim 9 \mu$ m-thick GaN layers grown by hydride vapor-phase epitaxy (HVPE) on sapphire $[16,17]$. The second set consisted of unintentionally doped n-type GaN layers grown by molecular beam epitaxy (MBE) on sapphire. The concentration $(n)$ of the majority carriers varied between $1 \times 10^{15}$ and $1 \times 10^{19} \mathrm{~cm}^{-3}$ and the Hall mobility $(\mu)$ between 9 and $100 \mathrm{~cm}^{2} / \mathrm{Vsec}$ respectively. The third set of samples consisted of unintentionally n-doped MBE-grown GaN layers with an AlN/GaN superstructure or with a 10 nm thick $\mathrm{Al}_{\mathrm{x}} \mathrm{Ga}_{1-\mathrm{x}} \mathrm{N}$ layer on the top.

The PEC etching was carried out in a standard electrochemical cell at room temperature using an unstirred $0.02 \mathrm{M} \mathrm{KOH}$ solution and a He-Cd laser $(\lambda=325 \mathrm{~nm})$ as the UV light source. A $100 \mathrm{~nm}$-thick Ti-mask was patterned around the periphery of the sample with a standard liftoff process. Additionally, the annealed Ti contact served to assist photo-current conduction. An amp-meter was used to monitor the photo-current that being proportional to the reaction rate at the semiconductor/electrolyte interface provides an instantaneous measure of the etch rate. No significant current was observed without sample illumination and consequently no etching was found in the regions of samples not subject to the laser illumination. For most of the $\mathrm{GaN}$ samples, no external bias was required in order to produce etching. However, for some highly resistive samples an additional bias was required between the sample and the cathode.

Characterization of the etched samples was carried out using a Digital Instruments tapping-mode AFM in both cross-sectional and plan-view configurations and a LEO 440 scanning electron microscope (SEM). Additionally, some as-grown samples were observed by plan-view TEM to estimate the dislocation density. 


\section{RESULTS AND DISCUSSION}

Slightly carrier-limited conditions with moderate illumination intensity $\left(\approx 100 \mathrm{~mW} / \mathrm{cm}^{2}\right)$ were found to etch crystalline GaN selectively, leaving vertical wires on the surface. In the AFM image of figure 1 is shown the surface morphology of the HVPE-grown sample after a 60 min PEC etching. The height of the whisker-like features is $\sim 700 \mathrm{~nm}$ and the diameter $\sim 100 \mathrm{~nm}$. The whisker density is about $1 \times 10^{9} \mathrm{~cm}^{-2}$. According to a previous TEM analysis performed on similar samples, this value is quite close to the effective density of dislocations [16]. Additionally, our plan-view TEM observations of as-grown samples revealed dislocation densities in the range $0.5-2 \times 10^{9} \mathrm{~cm}^{-2}$.

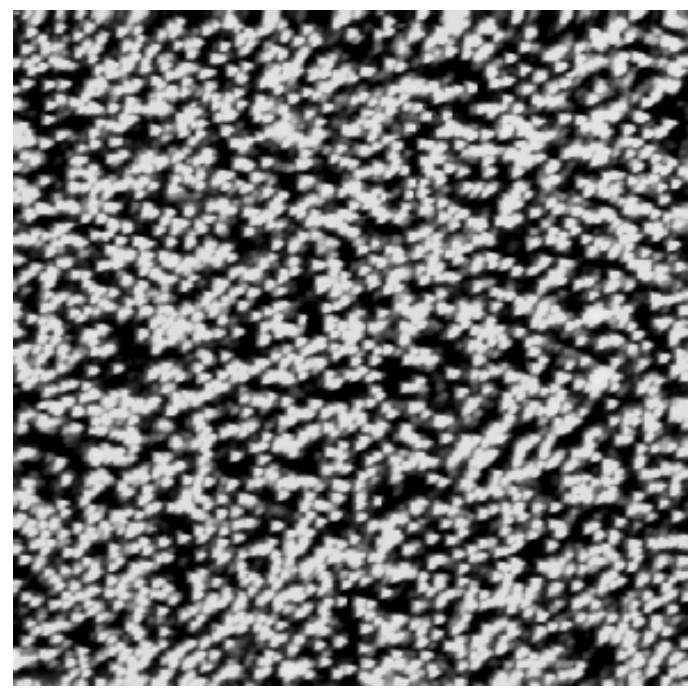

Figure 1 Tapping-mode AFM image $\left(15 \times 15 \mu \mathrm{m}^{2}\right)$ of the HVPE grown GaN sample etched by 60 min PEC process. Whisker-like features formed by etching are present on the surface. We estimate the height of these features to be $\sim 700 \mathrm{~nm}$ and the lateral size $\sim 100 \mathrm{~nm}$. The vertical scale ranges from 0 to $1200 \mathrm{~nm}$.

Using the same etching conditions for the MBE-grown samples, we have found a direct relation between the etch rate of $\mathrm{GaN}$ between dislocations (height of whiskers) and the electron concentration. We propose that the high etch rate in the $\mathrm{n}^{+}$-type $\mathrm{GaN}$ samples is due to better confinement of photogenerated holes at the surface, as previously reported for the PEC etching of $\mathrm{GaN}$ and GaAs $[18,19]$. Indeed, the Debye length is smaller for samples with higher doping, and assuming the same or similar values of band bending, the concentration of non-equilibrium holes at the surface should be higher for $\mathrm{n}^{+} \mathrm{GaN}$.

The AFM and SEM images of figure 2 show the etched surface morphology of the MBEgrown GaN layer with high electron concentration $\left(\mathrm{n} \approx 1 \times 10^{18} \mathrm{~cm}^{-3}\right)$. The 60 -min PEC process produces whisker-like features on the surface with a density of about $2 \times 10^{9} \mathrm{~cm}^{-2}$, as revealed by the AFM image of figure $2 \mathrm{a}$. The aspect ratio of these features is on the same order of magnitude as those found on HVPE-grown sample. For a better view, an AFM image was taken at roughly $15^{\circ}$ tilt with respect to horizontal (Fig. 2b). On the same etched sample after cleavage, we performed tapping-mode cross-sectional AFM measurements by tilting the sample by $\sim 90^{\circ}$ (Fig. 2c). Here clearly visible are nanometer-scale vertical wires on sapphire formed by etching selectively $\mathrm{GaN}$ between dislocation sites. The etched surface morphology was also investigated by SEM (Fig. 2d) The calculated density of the whisker-like features (white dots in figure) is $\approx$ $2 \times 10^{9} \mathrm{~cm}^{-2}$, the same value obtained from the AFM characterization. 


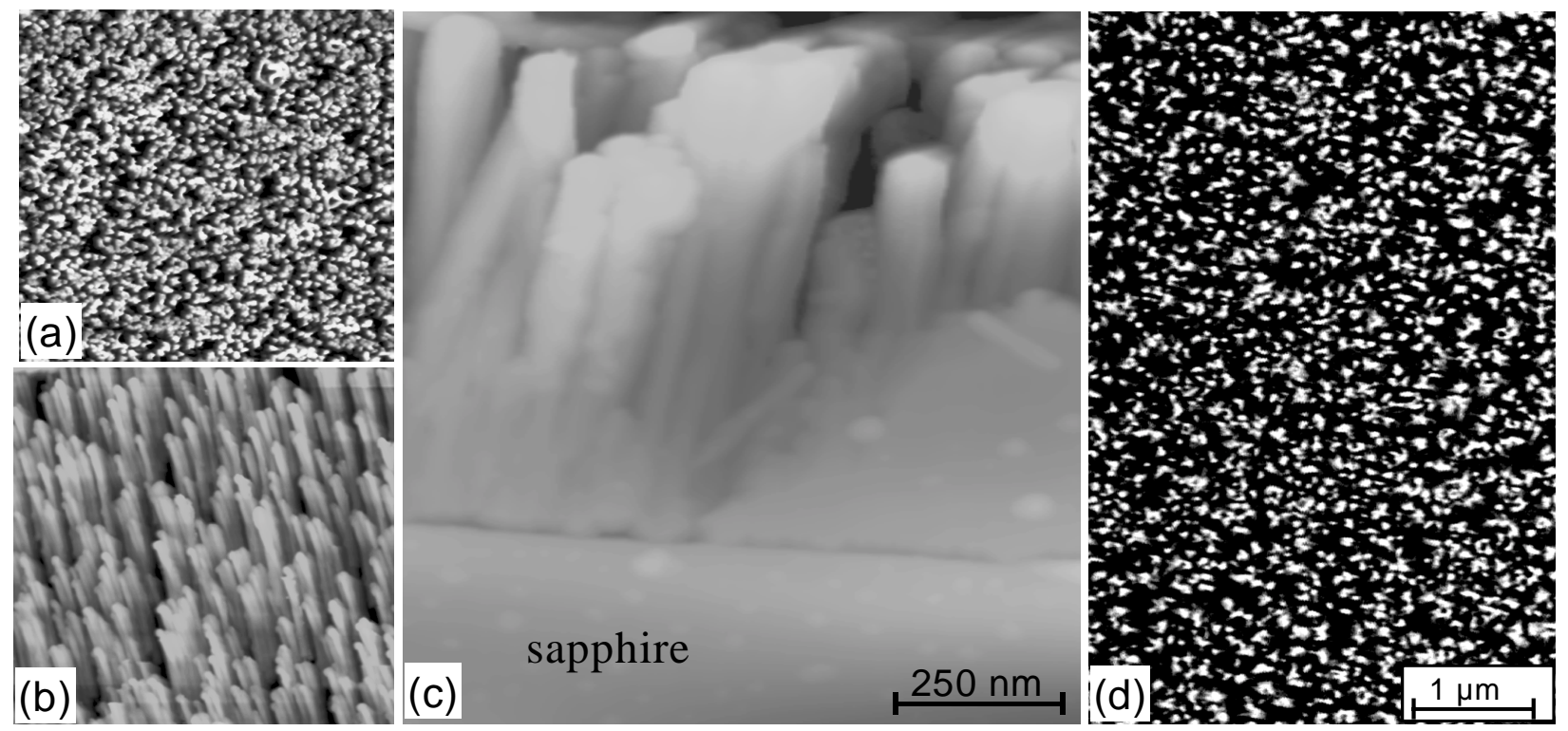

Figure 2 (a) AFM image (10x10 $\mu^{2}$ ) of the PEC etched surface morphology of the MBE-grown GaN layer. The whisker-like features formed on the surface have density of about $2 \times 10^{9} \mathrm{~cm}^{-2}$. (b) AFM image $\left(4 \times 4 \mu \mathrm{m}^{2}\right)$ of the same etched sample with tilting of $\sim 15^{\circ}$. Nanometer-scale threading wires are clearly visible. (c) Tapping-mode cross-sectional AFM image of the etched sample. The selective etching takes place in GaN producing vertical features on the sapphire surface. (d) Plan-view SEM image of the PEC etched sample. The density of whiskers (white dots) is $\sim 2 \times 10^{9} \mathrm{~cm}^{-2}$ the same value obtained from AFM.

Using higher illumination densities $\left(10 \mathrm{~W} / \mathrm{cm}^{2}\right)$ for MBE-grown GaN layers with high electron concentrations, we obtain a high etching rate $(\approx 0.1 \mu \mathrm{m} / \mathrm{min})$ with a smooth free-features etched surface. After 5 minutes of unbiased PEC etching, the root-mean-square roughness of the etched surface is comparable to that of the as-grown sample. The whisker formation by PEC etching is due to the rapid recombination at the dislocation sites of the photogenerated holes that thus cannot participate in the etching process. At high illumination densities, the holes are present near the surface in large excess because the etching rate is limited by the rate at which reactants in the solution diffuse and react with the semiconductor surface (strongly diffusionlimited condition) instead of the availability of holes. We suggest that this excess of holes saturates the defect states leading to etching also in the dislocation regions, in turn resulting in smooth etched surface.

We also carried out PEC etchings with moderate excitation intensity and the application of an external bias of both polarities between the sample (anode) and the wire (cathode). The application of a positive voltage to the MBE-grown samples with high electron concentration resulted in increased etch rate $(\approx 0.1 \mu \mathrm{m} / \mathrm{min}$ with a voltage of $+1 \mathrm{~V})$ with respect to the unbiased case and in smooth etched surfaces without whisker-like features. On the contrary, the application of a negative voltage to the sample sharply decreased the etch rate so much that at $-2 \mathrm{~V}$ we could not observe any appreciable etching in the illuminated region after several hours. The observed behavior may be attributed to increased band bending by the applied positive voltage which leads to better confinement of non-equilibrium holes near the surface and higher etch rates. The reason that the etched surface is smooth is similar to the case of high excitation density: the abundance of holes at the surface layer leads to the etching also at the dislocation sites. In case of negative bias, the band bending at the surface is reduced or even eliminated, leading to worse confinement or even surface depletion of the photogenerated holes. We repeated the above experiments for the sample with low electron concentration $\left(\mathrm{n} \approx 10^{16} \mathrm{~cm}^{-3}\right)$, 
for which no etching was revealed in the unbiased PEC process. We found that for negative and small positive voltages there was no etching, while some discernable etching was detected for large positive voltages. Similarly the photo-current remained close to zero up to a applied voltage of $+0.5 \mathrm{~V}$ and then started to rise. In particular, with a voltage of $+1.5 \mathrm{~V}$, the etch rate was about $10 \mathrm{~nm} / \mathrm{min}$ and the photo-current was $\sim 12 \mu \mathrm{A}$.

We also carried out PEC experiments on samples of different structure, for example, with an AlN/GaN superstructure below a $0.7 \mu \mathrm{m}$-thick GaN top layer. The etching process was stopped by the AlN layer, as also confirmed by measuring the depth of the etched region by a surface profilometer. Figure 3 a shows the structure of the sample after 40 minutes of etching. Note that the thickness of the etched GaN layer was $0.7 \mu \mathrm{m}$ and that of the AlN stop layer was only $\sim 5 \mathrm{~nm}$. The evolution of the photo-current during the etching process (Fig. 3b), shows that, after 30 minutes, the photo-current reduced to zero even though UV illumination was continued for an additional 10 minutes, demonstrating that the PEC etching stopped at the thin AlN layer. The high selectivity between AlN and GaN is due to the etching mechanism of the PEC process. Indeed, since the bandgap of AlN layer is larger than the excitation photon energy used, it is not possible to photogenerate electron-hole pairs on the semiconductor surface when the etching front reaches the AlN stop layer.
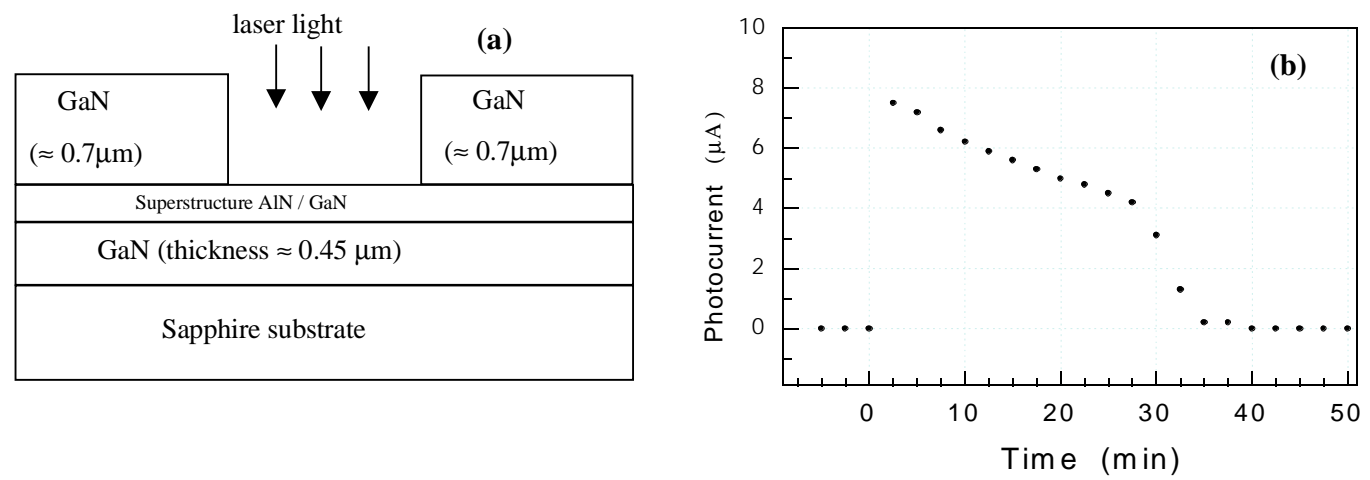

Figure 3 (a) Structure of the sample with superstructure AlN/GaN inside after 40 minutes of PEC process. The etching stopped at the AIN layer. (b) Time evolution of the photo-current during the PEC process.

Additionally, carrying out PEC experiments on samples with an $\mathrm{Al}_{\mathrm{x}} \mathrm{Ga}_{1-\mathrm{x}} \mathrm{N}$ layer on top, we observed that $\mathrm{Al}_{\mathrm{x}} \mathrm{Ga}_{1-\mathrm{x}} \mathrm{N}$ could be etched only if its bandgap is smaller than the excitation photon energy. In short, a $10 \mathrm{~nm}$ thick $\mathrm{Al}_{\mathrm{x}} \mathrm{Ga}_{1-\mathrm{x}} \mathrm{N}$ layer $(\mathrm{x}=5 \%)$, grown on thick $\mathrm{GaN}$, was easily etched, while similar layers with $x>20 \%$ remained unetched after several hours of PEC process. These results were confirmed by photoluminescence measurements carried out in unetched samples using the same He-Cd laser. We have observed the near-band-edge emission from the $\mathrm{AlGaN}$ layer in the first case, which confirms that the laser light has been absorbed in this layer because its bandgap was smaller than the photon energy. No such signal was detected in the second case $(x>20 \%)$ showing that no carriers could be photogenerated in the surface layer during the PEC process.

\section{CONCLUSIONS}

We have carried out PEC etching experiments on n-type MBE and HVPE-grown GaN samples in order to ascertain the feasibility of this method for dislocation-density estimation and device fabrication. Moderate illumination density was used to etch $\mathrm{GaN}$ between dislocation sites selectively, producing free-standing nanometer-scale vertical wires. Under these slightly 
carrier-limited conditions, the height of the revealed features was strongly dependent on the electron concentration of the GaN films. Using a higher illumination intensity or by applying an external single-polarity voltage, the PEC process became diffusion-limited, leading to high etch rates with smooth etched surfaces. In highly resistive samples for which, under normal etching conditions, the band bending was too small to confine the holes at the surface, the application of a single polarity voltage allowed etching to occur. Moreover, we have demonstrated high selectivity between $\mathrm{Al}_{\mathrm{x}} \mathrm{Ga}_{1-\mathrm{x}} \mathrm{N}(\mathrm{x}>20 \%)$ and $\mathrm{GaN}$ materials due to the etching mechanism of the PEC process. Indeed, if the bandgap of $\mathrm{Al}_{\mathrm{x}} \mathrm{Ga}_{1-\mathrm{x}} \mathrm{N}$ layer is larger that the excitation photon energy, the unavailability of photogenerated holes disables the PEC process.

\section{ACKNOWLEDGMENTS:}

The authors would like to thank Profs. A. Baski and K. J. Wynne for collaborations, L. Kerwath and J. Uilk for assistance in AFM and T. King for his tireless assistance throughout the laboratory. The VCU portion of this work was funded by grants from AFOSR (Dr. G. L. Witt), NSF (Drs. L. Hess and G. Pomrenke), and ONR (Drs. C. E. C. Wood and Y. S. Park). The Lincoln Laboratory portion of this work was sponsored by the Office of Naval Research under Air Force contract \#F19628-00-C-0002. Opinions, interpretations, conclusions and recommendations are those of the authors and not necessarily endorsed by the United States Air Force.

\section{REFERENCES}

[1] H. Morkoç, "Nitride Semiconductors and Devices," Springer Verlag, Heidelburg (1999).

[2] S. J. Pearton, J. C. Zolper, R. J. Shul, F. Ren, J. Appl. Phys. 86, 1, (1999).

[3] C. B. Vartuli, S. J. Pearton, C. R. Abernathy, R. J. Shul, A. J. Howard, S. P. Kilcoyne, J. E. Parmeter and M. Hagerott-Crawford, J. Vac. Sci. Technol. A, 14, 1011, (1996).

[4] M. E. Lin, Z. F. Fan, Z. Ma, L. H. Allen and H. Morkoç, Appl. Phys. Lett. 64, 887, (1994).

[5] C. B. Vartuli, J. D. MacKenzie, J. W. Lee, C. R. Abernathy, S. J. Pearton, R. J. Shul, J. Appl. Phys., 80, 3705, (1996).

[6] C. R. Eddy, MRS J. Nitride Semicond. Res. 4S1, G10.5 (1999).

[7] M. S. Minsky, M. White and E. L. Hu, Appl. Phys. Lett., 68, 1531, (1996).

[8] C. Youtsey, I. Adesida, G. Bulman, Appl. Phys. Lett., 71, 2151, (1997).

[9] C. Youtsey, I. Adesida, L. T. Romano, G. Bulman, Appl. Phys. Lett., 72, 560, (1998).

[10] L. H. Peng, C.-W. Chuang, J.-K. Ho, C.-N. Huang and C.-Y. Chen, Appl. Phys. Lett., 72, 939, (1998).

[11] D. A. Stocker, E. F. Schubert, K. S. Boutros, J. M. Redwing, MRS Int. J. Nitride Semicond. Res. 4S1, G7.5 (1999).

[12] C. Youtsey, L. T. Romano, I. Adesida, Appl. Phys. Lett. 73, 797, (1998).

[13] C. Youtsey, L. T. Romano, R. J. Molnar, I. Adesida, Appl. Phys. Lett. 74, 3537, (1999).

[14] I. Adesida, C. Youtsey, A. T. Ping, F. Khan, L. T. Romano and G. Bulman, MRS Int. J. Nitride Semicond. Res. 4S1, G1.4, 1999.

[15] N. G. Weimann, L. F. Eastman, D. Doppalapudi, H. M. Ng and T. D. Moustakas, J. Appl. Phys. 83, 3656, (1998).

[16] L. T. Romano, B. S. Krusor, R. J. Molnar, Appl. Phys. Lett. 71, 2283, (1997).

[17] W. Götz, L. T. Romano, B. S. Krusor, N. M. Johnson, R. J. Molnar, Appl. Phys. Lett. 69, 242, (1996).

[18] R. Khare and E. L. Hu, J. Electrochem. Soc., 138, 1516, (1991).

[19] M. N. Ruberto, X. Zhang, R. Scarmozzino, A. E. Willner, D. V. Podiesnik and R. M. Osgood Jr., J. Electrochem. Soc. 138, 1174, (1991). 